\title{
Genome-wide analysis of Dongxiang wild rice (Oryza rufipogon Griff.) to investigate lost/acquired genes during rice domestication
}

Fantao Zhang ${ }^{1 \dagger}$, Tao Xu$^{2+}$, Linyong Mao ${ }^{3}$, Shuangyong Yan ${ }^{4}$, Xiwen Chen ${ }^{5}$, Zhenfeng $\mathrm{Wu}^{6}$, Rui Chen ${ }^{7}$, Xiangdong Luo ${ }^{1}$, Jiankun Xie ${ }^{1 *}$ and Shan Gao ${ }^{5^{*}}$

\begin{abstract}
Background: It is widely accepted that cultivated rice (Oryza sativa L.) was domesticated from common wild rice (Oryza rufipogon Griff.). Compared to other studies which concentrate on rice origin, this study is to genetically elucidate the substantially phenotypic and physiological changes from wild rice to cultivated rice at the whole genome level.

Results: Instead of comparing two assembled genomes, this study directly compared the Dongxiang wild rice (DXWR) Illumina sequencing reads with the Nipponbare (O. sativa) complete genome without assembly of the DXWR genome. Based on the results from the comparative genomics analysis, structural variations (SVs) between DXWR and Nipponbare were determined to locate deleted genes which could have been acquired by Nipponbare during rice domestication. To overcome the limit of the SV detection, the DXWR transcriptome was also sequenced and compared with the Nipponbare transcriptome to discover the genes which could have been lost in DXWR during domestication. Both 1591 Nipponbare-acquired genes and 206 DXWR-lost transcripts were further analyzed using annotations from multiple sources. The NGS data are available in the NCBI SRA database with ID SRP070627.

Conclusions: These results help better understanding the domestication from wild rice to cultivated rice at the whole genome level and provide a genomic data resource for rice genetic research or breeding. One finding confirmed transposable elements contribute greatly to the genome evolution from wild rice to cultivated rice. Another finding suggested the photophosphorylation and oxidative phosphorylation system in cultivated rice could have adapted to environmental changes simultaneously during domestication.
\end{abstract}

Keywords: Dongxiang wild rice, Whole genome sequencing, Transcriptome, Comparative genomics analysis, Structural variation

\section{Background}

Cultivated rice (Oryza sativa L.), as one of the most important agricultural crops, supplies the main dietary source for more than half of the world's population [1]. Although it is well accepted that cultivated rice was domesticated from common wild rice (Oryza rufipogon

\footnotetext{
* Correspondence: xiejiankun11@163.com; gao_shan@mail.nankai.edu.cn ${ }^{\dagger}$ Equal contributors

${ }^{1}$ College of Life Sciences, Jiangxi Normal University, Nanchang, Jiangxi 330022, P. R. China

${ }^{5}$ College of Life Sciences, Nankai University, Tianjin 300071, P. R. China Full list of author information is available at the end of the article
}

Griff.) thousands of years ago [2], the origin and domestication process of cultivated rice have been debated for decades through different studies [3-5]. Until recently, it was revealed that $O$. sativa $\mathrm{L}$. ssp. japonica had been first domesticated from a specific population of O. rufipogon around the middle area of the Pearl river in southern China, and that O. sativa L. ssp. indica had been subsequently developed from crosses between japonica and local wild rice as the initial cultivars spread into Southeast and South Asia [6]. Along with a multitude of studies on rice origin, further work is needed to 
genetically elucidate the substantially phenotypic and physiological changes from wild rice to cultivated rice at the whole genome level.

This study conducted comparative genomics analysis between $O$. sativa L. spp. japonica var. Nipponbare and Dongxiang wild rice (DXWR), a Chinese common wild rice (O. rufipogon). DXWR was firstly discovered in Dongxiang county, Jiangxi province of China in 1978 [7], which was considered as the most northern one $\left(28^{\circ}\right.$ 14 ' $\mathrm{N}$ ) of the regions where common wild rice populations had been discovered around the world. During the past three decades, DXWR has been well investigated as a precious genetic resource for cultivated rice improvement or fundamental research on genetic diversity $[8,9]$, heterosis [10], cytoplasmic male sterility [11], fertility restoration [12], biomass [13], high yield [14-16], and resistance to biotic and abiotic stress [17-21].

To perform the comparative genomics analysis, we sequenced the whole genome of DXWR using NextGeneration Sequencing (NGS) technologies. In this study, the strategy of the comparative genomics analysis was to directly compare the DXWR NGS reads with the Nipponbare complete genome without assembly of the DXWR genome. This strategy avoided the highly timeconsuming work and a substantial number of errors resulted from the de novo assembly of the DXWR genome using the NGS short reads. The essential work in this comparative strategy was using the software SVDetect and the pipeline SVFilter to detect structure variations (SVs), which are being increasingly appreciated for their roles as a cause for phenotypic variations [22-24]. Using the detected deletions (one important type of SVs), we located genes which could have been acquired by Nipponbare during rice domestication. To overcome the limit of the SV detection, the DXWR transcriptome was also sequenced and compared with the Nipponbare transcriptome to discover the genes which could have been lost in DXWR during domestication. Both Nipponbareacquired genes and DXWR-lost transcripts were further analyzed using annotations from multiple sources (e.g. QTLs for traits and KEGG pathways) to reach two research goals: 1) to help better understanding the domestication from wild rice to cultivated rice at the whole genome level; 2) to provide a genomic data resource for rice genetic research or breeding.

\section{Results and discussion}

\section{Whole-genome sequencing of Dongxiang wild rice}

The sequencing of the Dongxiang wild rice (DXWR) genome produced a total of 282,383,842 paired-end 90 bp cleaned reads (25.4 Gb data) using Illumina sequencing technology, covering 68 -fold of the reference genome (O. sativa L. spp. japonica var. Nipponbare) with the size $373,245,519$ bp. The high depth of this
Next-Generation Sequencing (NGS) data satisfied the requirement for the reliable detection of structure variations (SVs). Then, we mapped all the cleaned reads to nine complete rice genomes (Methods). Using singleend alignment of forward-sequenced reads, the rate of mapped reads against the total reads reached 74.19, $56.98,47.11,36.29,32.02,29.30,19.76,4.20$ and $1.18 \%$ for O. sativa L. spp. japonica var. Nipponbare, O. sativa L. spp. indica, O. nivara, O. glaberrima, O. barthii, $O$. glumaepatula, O. meridionalis, O. punctata and O. brachyantha, respectively. Nipponbare reached the highest mapped rate probably due to two reasons. The first reason is that $O$. sativa is considered to have been domesticated from Chinese common wild rice [2]. The second reason is that the Nipponbare genome was sequenced using the clone-by-clone approach with Sanger sequencing technology and is ranked as the best assembled and annotated one out of all rice genomes. Therefore, we used the Nipponbare genome as reference to detect SVs.

\section{Structural variations between Dongxiang wild rice and Nipponbare genome}

We used the software SVDetect to detect SVs between DXWR paired-end reads and the Nipponbare genome without assembly of the DXWR genome. The basic theory of SVDetect is to use priori information from paired-ends such as order, orientation and insert size of pairs (500 bp in this study) to classify mapped reads into normally and anomalously paired-end reads. Removing normally mapped paired-end reads, SVDetect uses anomalously mapped paired-end reads to produce SVs. Since SVDetect produces a large number of false positive SVs, we developed a pipeline named SVFilter to reduce the false positives. SVFilter uses five independent programs (ratiofilter, gapfilter, SNVfilter, coveragefilter and depthfilter) to successively filter out false positives (Methods). In this study, SVDetect produced 13,767 potential SVs and the ratiofilter largely reduced the SV number to 3946 (28.66\% of 13,767). Then, the gapfilter, SNVfilter, coveragefilter and depthfilter narrowed down the SV number to 3945, 3524, 2570 and 2539 (Additional file 1), respectively.

After removing the larger deletions which overlapped the smaller inside deletions, 1568 out of 1571 deletions were left for further analysis. Finally, 2536 SVs were determined to include 1568 deletions, 437 translocations, 423 inverted translocations, 88 inversions, six inverted duplications, three inverted fragment inversions, one fragment insertion and 10 undefined SVs. Among eight types of SVs, the deletions contribute to $61.83 \%$ (1568/ 2536) of the total SVs (Fig. 1a), followed by translocations/inverted translocations accounting for $33.91 \%$ (860/2536) of the total SVs (Fig. 1b). Generally speaking, the deletion number and the translocation/inverted 


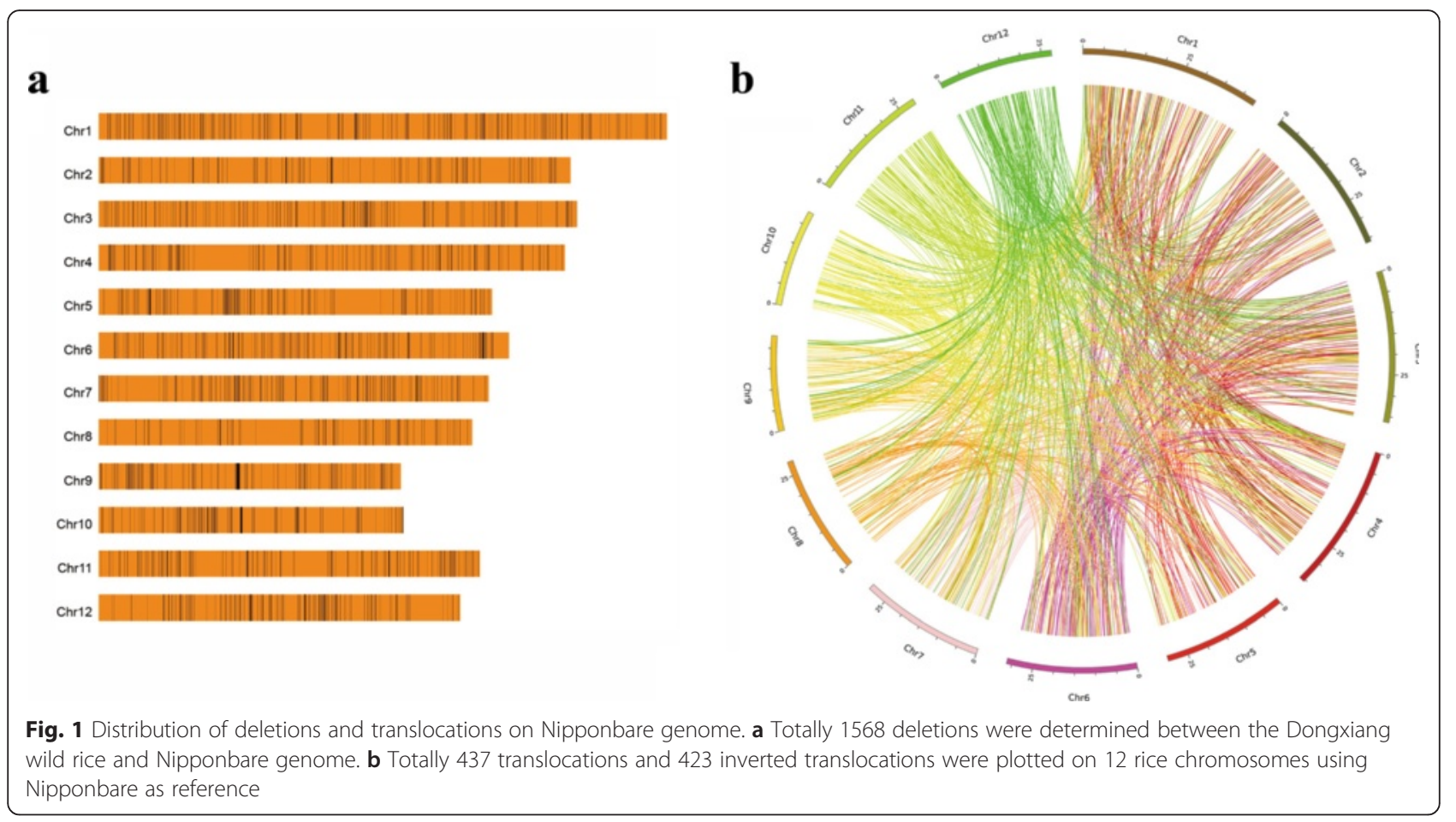

translocation number has a linear relationship with the chromosome length on a logarithmic scale, respectively (Fig. 2a). All chromosomes of the Nipponbare genome contain the deletions and translocations/inverted translocations in the same pattern with the exception of chromosome 9 . Moreover, chromosome 1 contains more deletions and translocations/inverted translocations than the other 11 chromosomes.

\section{Discovery of Nipponbare-acquired genes during rice domestication}

The SV detection and filtering process determined 1568 deletions. These deleted regions could contain genes which were hypothesized to have been acquired by Nipponbare during rice domestication. Mapping the 1568 deletions to rice genes in the Nipponbare genome, $61.47 \%(964 / 1568)$ of the total deletions were associated to 1591 genes (Additional file 2). Among 964 deletions, $67.32 \%$ (649/964) contain only one gene. The top eight deletions containing more than ten genes were mapped to the genomic region from $10790002 \mathrm{bp}$ to 10920963 bp on chromosome 10 (Chr10:1079000210920963), Chr9:10545593-10732410, Chr8:92427229284595, Chr2:14239399-14298646, Chr6:2356329423592857, Chr6:29241098-29330521, Chr2:1768169117753826 and Chr11:11280103-11340819 (Fig. 2b). Among 1591 Nipponbare-acquired genes, $77.18 \%$ $(1228 / 1591)$ are transposable elements (TEs), $60.99 \%$ (749/1228) of which are retrotransposons (Fig. 2c). In the total 55,801 Nipponbare genes, $30.34 \%(16,932 /$
$55,801)$ are TEs, $70.97 \%(12,017 / 16,932)$ of which are retrotransposons. More than two-fold (77.18 vs. $30.34 \%$ ) difference of TE percentages is consistent with the previous finding that TEs contribute greatly to the genome evolution from wild rice to cultivated rice [25].

Quantitative Trait Loci (QTLs) link particular regions on the genome to the agronomic traits. In rice, numerous QTLs for important agronomic traits have been identified and included in the Gramene QTL database (Methods). Mapping the deletions to the annotated rice QTLs in the Nipponbare genome, 731 QTLs located in 539 unique regions on the Nipponbare genome were associated to 937 deletions (Additional file 3). The phenotypes of these 731 QTLs belong to six trait categories. They are yield (216 of 731), vigor (197), anatomy (192), quality (51), biochemical (38) and development (37) (Fig. 2b). Combining the results from the previous steps, we constructed the relationship between QTLs and genes in the same regions affected by the deletions (Additional file 4). Finally, the relationship between 937 deletions, 1547 deleted genes and 731 QTLs was constructed for further studies (Fig. 2b). Using this relationship information, agronomic traits, QTLs and associated genes were summarized to help better understanding the domestication from wild rice to cultivated rice. During this domestication process, cultivated rice could have acquired genes involving plant height, spikelet number, panicle number, leaf senescence and panicle length on the top five traits, 


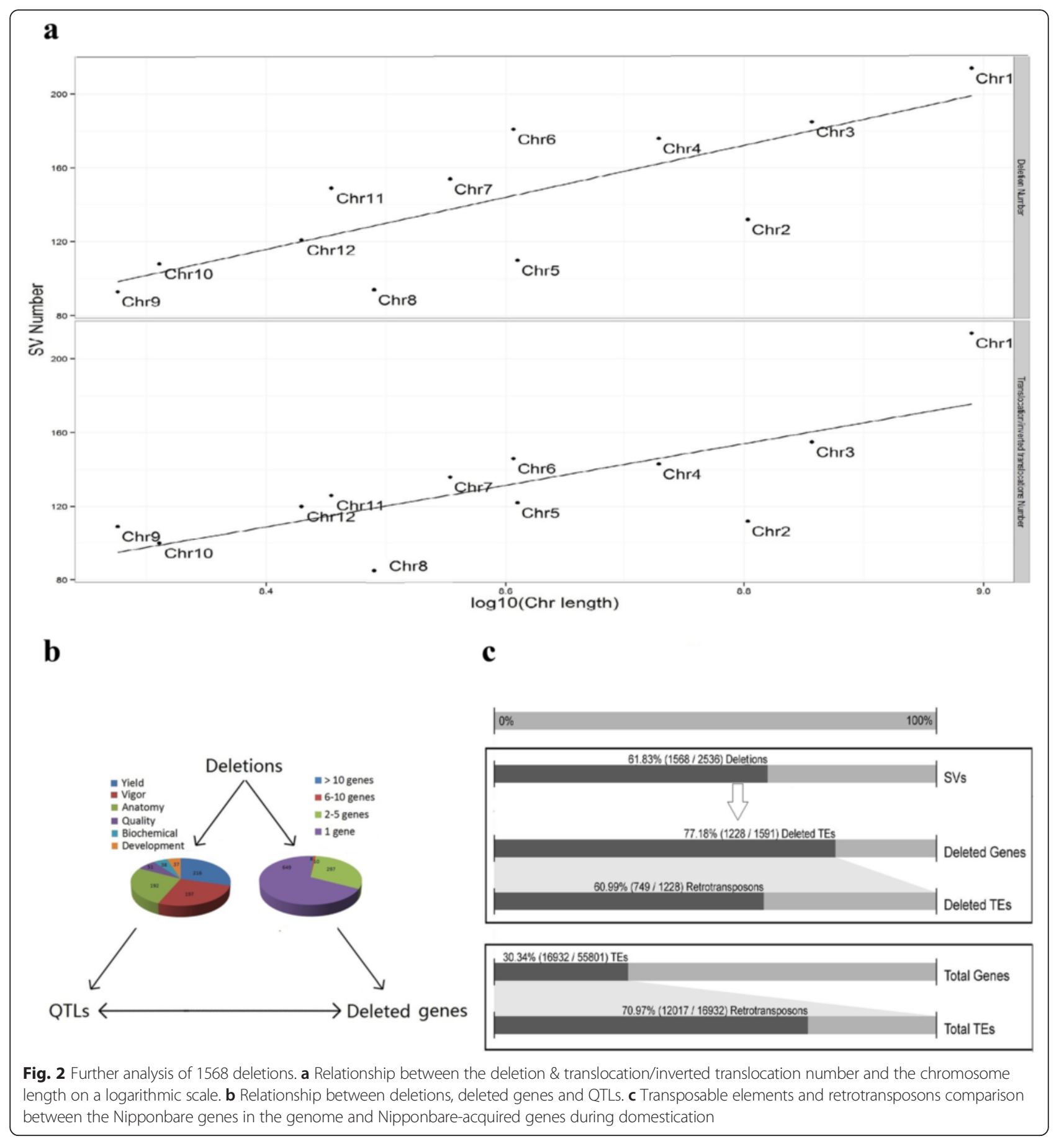

followed by biomass yield, seedling vigor, leaf width, tiller number and various other traits (Table 1).

\section{Discovery of DXWR-lost genes using the DXWR transcriptome}

In this study, we only used 500 bp paired-end reads from the DXWR genome for SV detection. This single library size limited the size of detected insertions on the DXWR genome to not more than $300 \mathrm{bp}$. Then, we could not detect large insertions ( $>300 \mathrm{bp}$ ) to locate DXWR-lost genes during rice domestication. Therefore, we sequenced and assembled the DXWR transcriptome to discover DXWR-lost genes. The total RNA was extracted from seedling leaves and seedling roots to construct two separate RNA-seq libraries, which were sequenced on the Illumina HiSeq 2000 system. After data cleaning and quality control, a total of 86,340,332 paired-end 100 bp raw reads (8.6 Gbp data) were 
Table 1 QTLs for traits and associated genes

\begin{tabular}{|c|c|c|}
\hline Trait & QTL number & Gene number \\
\hline Plant height & 86 & 1338 \\
\hline Spikelet number & 71 & 1019 \\
\hline Panicle number & 39 & 460 \\
\hline Leaf senescence & 37 & 669 \\
\hline Panicle length & 37 & 772 \\
\hline Biomass yield & 33 & 442 \\
\hline Seedling vigor & 32 & 416 \\
\hline Leaf width & 25 & 456 \\
\hline Tiller number & 25 & 434 \\
\hline 1000-seed weight & 22 & 504 \\
\hline Root number & 21 & 459 \\
\hline Root thickness & 20 & 285 \\
\hline Seed dormancy & 20 & 299 \\
\hline Chlorophyll content & 17 & 271 \\
\hline Leaf length & 17 & 324 \\
\hline Spikelet density & 16 & 472 \\
\hline Root length & 15 & 237 \\
\hline Seed number & 15 & 545 \\
\hline Anther length & 9 & 257 \\
\hline Culm thickness & 9 & 336 \\
\hline Awn length & 8 & 168 \\
\hline Culm length & 8 & 253 \\
\hline Seed length & 8 & 138 \\
\hline Grain yield & 7 & 71 \\
\hline Mesocotyl length & 7 & 67 \\
\hline Yield & 7 & 264 \\
\hline Carbohydrate content & 6 & 124 \\
\hline Carbon content & 6 & 184 \\
\hline Grain length & 6 & 113 \\
\hline Grain number & 6 & 199 \\
\hline Panicle weight & 6 & 296 \\
\hline Primary branch & 6 & 70 \\
\hline Root activity & 6 & 48 \\
\hline Flour color & 5 & 78 \\
\hline Leaf area & 5 & 84 \\
\hline Seed width & 5 & 110 \\
\hline 100-seed weight & 4 & 148 \\
\hline Germination speed & 4 & 35 \\
\hline Grain weight & 4 & 45 \\
\hline Head rice & 4 & 91 \\
\hline Amylose content & 3 & 16 \\
\hline Consistency viscosity & 3 & 78 \\
\hline Gelatinization temperature & 3 & 43 \\
\hline Grain shattering & 3 & 82 \\
\hline
\end{tabular}

Table 1 QTLs for traits and associated genes (Continued)

\begin{tabular}{lll}
\hline Grain width & 3 & 21 \\
Ratooning ability & 3 & 15 \\
Rubisco content & 3 & 16 \\
Secondary branch & 3 & 69 \\
Spikelet weight & 3 & 13 \\
Chlorophyll ratio & 2 & 25 \\
Flower number & 2 & 12 \\
Gel consistency & 2 & 10 \\
H2O2 content & 2 & 18 \\
Leaf height & 2 & 82 \\
Setback & 2 & 12 \\
Breakdown viscosity & 1 & 5 \\
Groat percentage & 1 & 51 \\
Leaf perimeter & 1 & 5 \\
Photosynthetic ability & 1 & 21 \\
Protein content & 1 & 4 \\
Root volume & 1 & 27 \\
Seed density & 1 & 15 \\
Seed weight & 1 & 12
\end{tabular}

The records were sorted by the QTL number. Trait used annotations from the Gramene database v40. Additional file 4 records the relationship between QTLs and rice genes in the same region on the reference chromosome

processed to $85,813,832$ cleaned reads, with the Q20 percentage of $99.3 \%$. These cleaned reads were de novo assembled into the DXWR transcriptome, filtering out contigs shorter than $200 \mathrm{bp}$.

The DXWR transcriptome contains 70,747 genes and 99,092 transcripts with the average length $968 \mathrm{bp}$ and the N50 length $1655 \mathrm{bp}$, while the Nipponbare transcriptome contains 55,204 genes and 65,556 transcripts with the average length $1722 \mathrm{bp}$ and the N50 length $2295 \mathrm{bp}$, filtering out the transcripts shorter than $200 \mathrm{bp}$. The length distribution of the assembled DXWR transcriptome was compared with the length distribution of the Nipponbare transcriptome (Fig. 3a). From Fig. 3a, it can be seen the number of DXWR and Nipponbare transcripts decreases with the transcript length in a similar pattern, with an exception of transcripts shorter than 1000 bp. Particularly, DXWR has more short transcripts (<500 bp) than Nipponbare does. This difference is mainly due to the incomplete assembly of these DXWR transcripts using the NGS short reads. Although 45,737 (46.16\% of 99,092) DXWR transcripts have lengths less than $500 \mathrm{bp}, 53,355(53.84 \%$ of 99,092$)$ transcripts with lengths at least 500 bp still provide abundant information for further studies. In addition, Nipponbare has more high-GC transcripts than DXWR does (Fig. 3b). The previous study indicated that high-GC genes in rice and other cereals have greater bias and codon usage is 

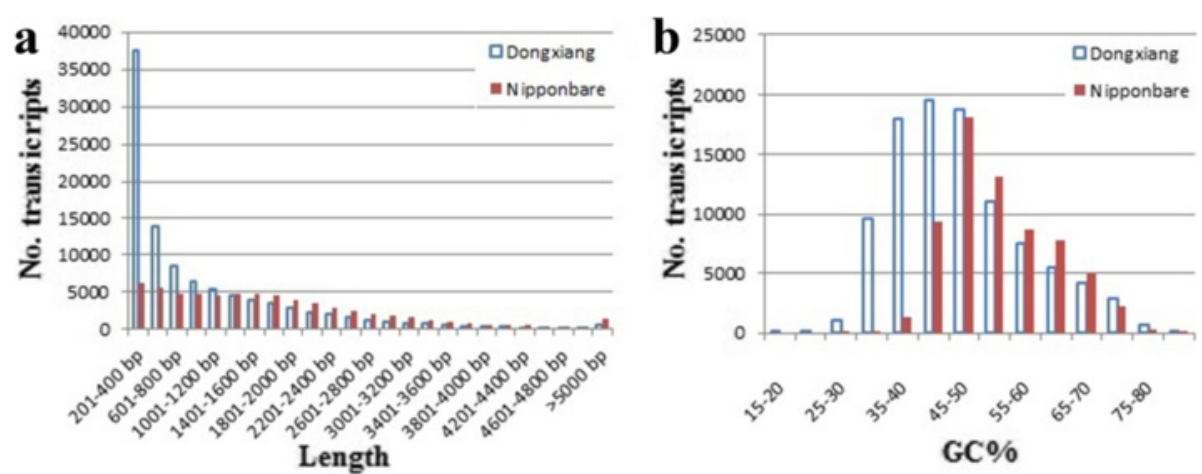

Fig. 3 Dongxiang wild rice and Nipponbare transcriptome. Dongxiang and Nipponbare represents the Dongxiang wild rice and Nipponbare transcripts longer than $200 \mathrm{bp}$. The Dongxiang transcriptome could contain transcripts from chloroplast or mitochondria. a Length distribution. b GC-content distribution

skewed toward codons that are preferred in highly expressed genes [26].

Using the program blastx, 60,247 (60.8\% of 99,092) transcripts were annotated by the functional description of their top 20 similar sequences (hits), if they existed, from the NCBI Non-Redundant Protein (NR) database (Additional file 5). Using the software blast2go, 35,596 $(35.92 \%$ of 99,092$)$ transcripts were annotated by Gene Ontology (GO) slim terms for plants in three domains, molecular function, cellular component and biological process. By mapping GO slim terms to enzyme codes and the Kyoto Encyclopedia of Genes and Genomes (KEGG) database, 4548 (4.59\% of 99,092) transcripts were assigned to 137 KEGG pathways (Additional file 6). Using the program blastn, 99,092 DXWR transcripts were mapped to the Nipponbare transcriptome and then, 409 unmapped transcripts were mapped to the Nipponbare mitochondrial cDNA, chloroplast cDNA and nuclear genome sequences. Finally, 206 transcripts from 205 genes were identified as DXWR-lost transcripts during domestication.

\section{Further analysis of Nipponbare-acquired and DXWR-lost genes}

To further analyze 1591 Nipponbare-acquired genes, 1652 putative proteins encoded by them were mapped to KEGG pathways, known transcription factors (TFs) and protein kinases (PKs), respectively. The significant results included 13 genes involving the photosynthesis pathway (Fig. 4), 11 genes involving the oxidative phosphorylation pathway (Fig. 5), three putative proteins encoded by two genes (LOC_Os08g33488 and LOC_Os12g31748) belonging to the transcription factor MIKC, three putative proteins encoded by three genes (LOC_Os09g29540, LOC_Os09g29560 and LOC_Os09g29584) belonging to the wall associated kinase (WAK) or wall associated kinase-like kinase family, seven putative proteins encoded by the gene LOC_Os01g20900 belonging to the receptor like cytoplasmic kinase VIII family and five putative proteins encoded by the gene LOC_OsO1g27020 belonging to the CDC2 like kinase family. Further analysis showed 18 of 1591 Nipponbare-acquired genes have only one copy in the genome. These 18 genes encode proteins from $\mathrm{HNH}$ endonuclease family, trehalose phosphatase, MYB family transcription factor, ubiquitin-protein ligase 1 and peptide transporter PTR2, et al. (Additional file 7). Further analysis of 206 DXWR-lost transcripts showed 18 of them had functional annotations and three of them were enriched in the oxidative phosphorylation pathway. These three transcripts encode three proteins, which are cytochrome b, cytochrome oxidase subunit I and NADdependent aldehyde dehydrogenase (Additional file 7).

MIKC as one type of MADS-box TFs has been implicated in several aspects of plant growth and development. Genome-wide identification and phylogenetic analyses of 75 MADS-box genes in rice showed: 1) the Nipponbare-acquired gene LOC_Os08g33488 (OsMADS23) and LOC_Os12g31748 (OsMADS20) was categorized into the AGL17-like and the SQUA-like group, respectively; 2) these two MADS-box genes were genetically far from other genes within their groups [27]. The WAK gene family plays important roles in cell expansion, pathogen resistance and heavy-metal stress tolerance in Arabidopsis. The OsWAK gene family containing 125 members expanded in the rice genomes due to lineage-specific expansion of the family in monocots [28]. The Nipponbare-acquired gene LOC_Os09g29540 (OsWAK82), LOC_Os09g29560 (OsWAK83) and LOC_Os09g29584 (OsWAK84) was classified into the type of OsWAKRLCK, OsWAK pseudogene and OsWAK-RLP [28], respectively.

In total, 13 Nipponbare-acquired genes were enriched in the photosynthesis pathway, 11 Nipponbare-acquired genes and three DXWR-lost transcripts were enriched in the oxidative phosphorylation pathway (Additional file 


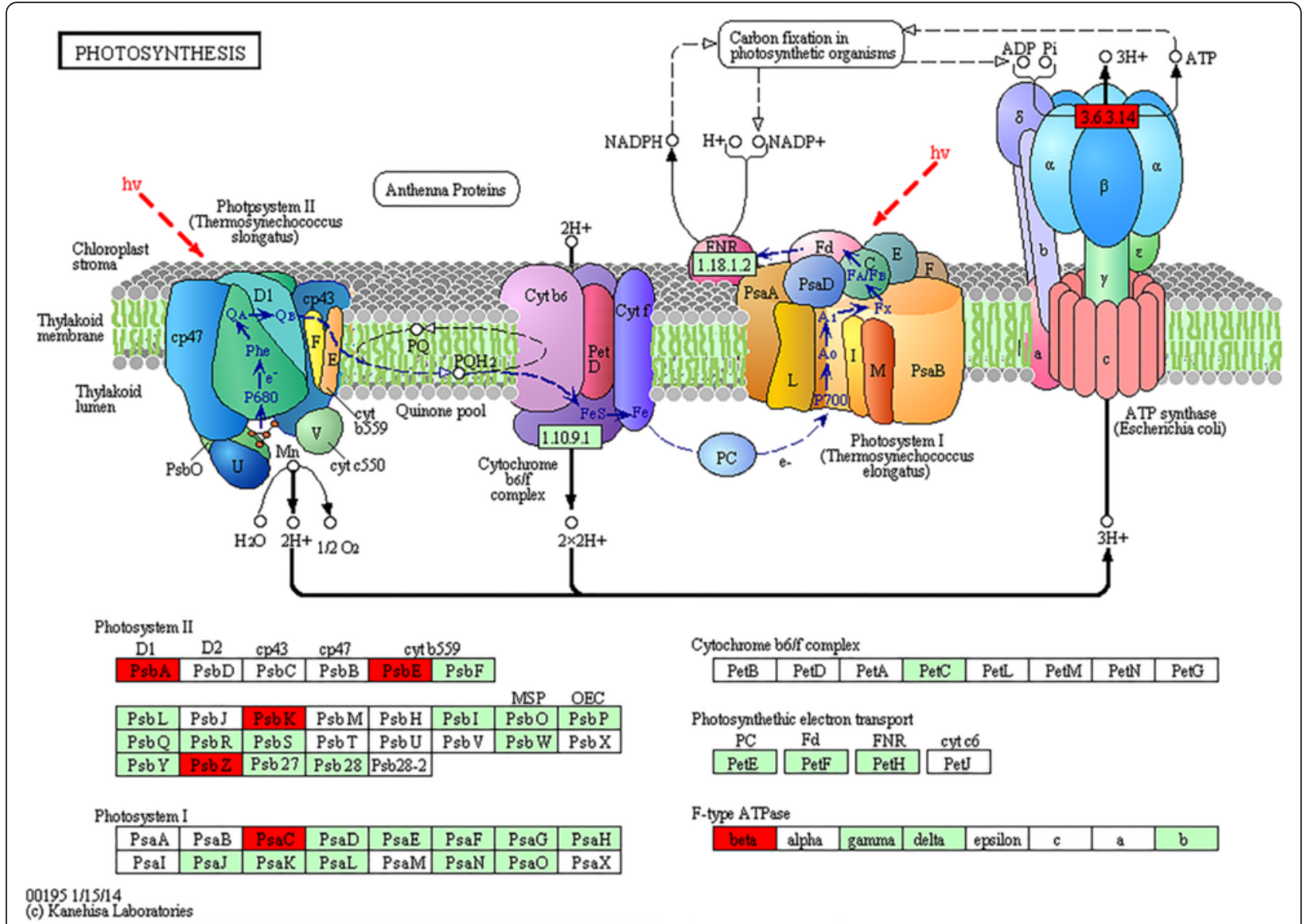

Fig. 4 The photosynthesis pathway with Nipponbare-acquired genes. This figure (KEGG: map00195) was downloaded from the KEGG website with copy-right permission. Among 1591 Nipponbare-acquired genes, 13 photosynthesis related genes were mapped to six proteins (Additional file 7: Table S2). They are photosystem II PsbA, photosystem II PsbE, photosystem II PsbK, photosystem II PsbZ, photosystem I PsaC and F-type ATPase beta (in red)

7). Both photophosphorylation and oxidative phosphorylation are potential ATP sources for the plasmalemma ATPase, which drives the phosphorylation reaction to releases energy. Previous studies showed these two energy systems cooperate to provide energy. One example is mitochondrial oxidative phosphorylation appears to serve an essential function for supplying the cytosol with ATP during photosynthesis [29]. Another example is photophosphorylation in the light and oxidative phosphorylation in the dark supply energy together for the opening of stomata on the epidermal peels of Commelina communis L. and Vicia faba L. [30]. Previous studies also showed that cultivated rice possesses higher photosynthetic efficiency than wild rice does to produce high yield [31, 32]. These findings together suggest the photophosphorylation and oxidative phosphorylation system in rice could have adapted to environmental changes simultaneously during domestication from wild rice to cultivated rice.

\section{Conclusions}

In this study, we performed the whole genome sequencing of Dongxiang wild rice (DXWR), a Chinese common wild rice. Using the software SVDetect and the pipeline SVFilter, 2536 structural variations (SVs) were determined between DXWR and the reference genome Nipponbare. Out of 2536 SVs, 1568 deletions were used to locate 1591 deleted genes, which were hypothesized to have been acquired by Nipponbare during its domestication. To overcome the DNA library size limit, the DXWR transcriptome was sequenced and compared with the Nipponbare transcriptome to obtain 206 DXWR-lost transcripts during rice domestication. Further analysis of Nipponbare-acquired genes and DXWRlost transcripts showed 13 Nipponbare-acquired genes were enriched in the photosynthesis pathway. In addition, 11 Nipponbare-acquired genes and three DXWR-lost transcripts were enriched in the oxidative phosphorylation pathway. The photophosphorylation and oxidative phosphorylation system in rice could have 


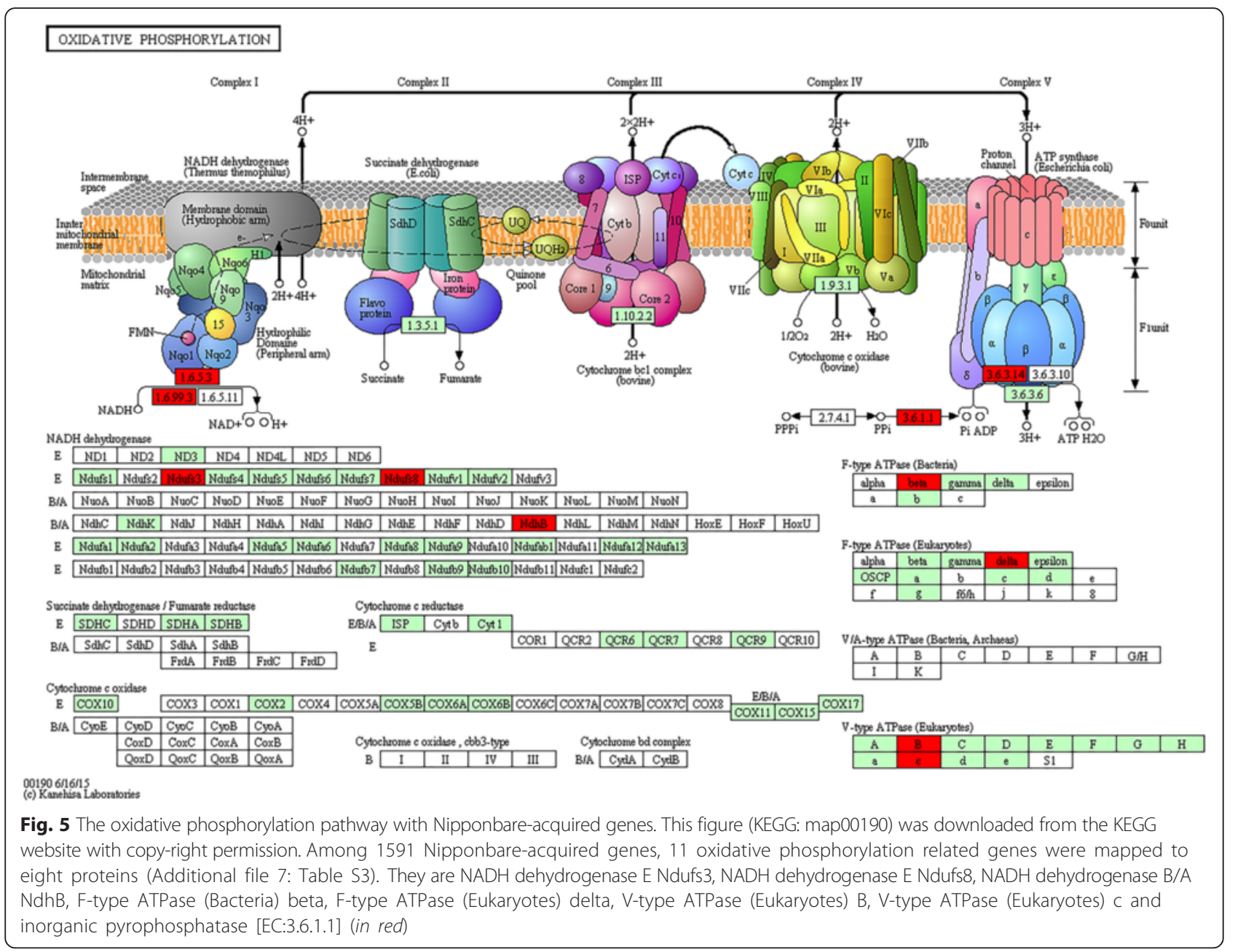

adapted to environmental changes simultaneously during domestication from wild rice to cultivated rice.

In this study, the relationship between 937 deletions, 1547 deleted genes and 731 QTLs was constructed to provide a valuable resource for further studies. Using this relationship information, agronomic traits, QTLs and associated genes were summarized to help better understand the substantially phenotypic and physiological changes from wild rice to cultivated rice at the whole genome level. This information can also be used to guide the future experiments for rice genetic research or breeding.

\section{Methods}

\section{DNA and RNA sequencing of Dongxiang wild rice}

Dongxiang wild rice (DXWR) is ex situ conserved in Jiangxi Academy of Agricultural Sciences, Nanchang, China (http://www.jxaas.com/index.html), and the seeds of DXWR are freely available for scientific research. In order to avoid the interference from extraneous pollen and reduce the heterozygosity, DXWR had been subjected to self-pollination by bagging cultivation for more than ten years. The seeds used in this study were acquired with a Material Transfer Agreement (MTA) from the Jiangxi Academy of Agricultural Sciences. Firstly, the seeds of DXWR were growing under natural conditions in a paddy field at the experimental station. Then, the DXWR seeds from the paddy field were planted in the growth chamber $\left(28 \pm 2{ }^{\circ} \mathrm{C}, 14 \mathrm{~h} /\right.$ day and $10 \mathrm{~h} /$ night $)$ to acquire seedlings for DNA and RNA extraction. The experimental research reported here complies with institutional, national, and international guidelines concerning plant genetic repositories.

The DNA was extracted from the seedling leaves and the total RNA was from seedling leaves and seedling roots at the four-leaf stage, separately. The DNA-seq library with the $500 \mathrm{bp}$ insert size was constructed using Illumina TruSeq DNA Sample Prep Kit and sequenced using the $90 \mathrm{bp}$ paired-end technology on the Illumina HiSeq 2000 system. Two non-strand-specific RNA-seq libraries with the $200 \mathrm{bp}$ insert size were constructed using Illumina TruSeq RNA Sample Prep Kit and sequenced using the $100 \mathrm{bp}$ paired-end technology on the Illumina HiSeq 2000 system. The cleaning and quality 
control of the DNA-seq and RNA-seq data were conducted using the pipeline Fastq_clean [33] that is optimized to clean the raw reads from Illumina platforms [34-41]. Low quality $(<$ Q20) and adapter contained reads were removed from the DNA-seq data. As for the RNA-seq data processing, low quality $(<$ Q20) nucleotides on both ends of the raw reads were trimmed and the trimmed reads which contain ambiguous nucleotides ("N") at least two were removed. Then, adapter segments on the 3' ends of the remaining reads were trimmed and short $(<25 \mathrm{bp})$ trimmed reads were removed. Finally, the virus-like and rRNA-like RNA-seq reads were removed.

\section{Rice genomes, MSU annotation and QTL data}

Nine complete genome data were downloaded from the Ensembl database v22 (ftp://ftp.ensemblgenomes.org/ pub/release-22/plants). The sequences with annotation of the reference genome (O. sativa L. spp. japonica var. Nipponbare) in Ensembl v22 are identical to the data in IRGSP v1.0 and MSU_osalr v7.0 (MSU Rice Genome Annotation Project Release 7). The gene and protein annotation used MSU_osalr v7.0 (http://rice.plantbiology.msu.edu/). The rice QTL data were downloaded from the Gramene database v40 (http://www.gramene.org) [42]. After redundancy removal, the number of QTL records was narrowed down from 8216 to 5165 .

\section{Software and programs}

The software Trinity r20140717 [43] was used to assemble the DXWR transcriptome. The software bowtie v0.12.7 [44] was used to align the cleaned DNA reads to nine complete rice genomes allowing two mismatches. The software bwa v0.5.7-r1310 was used to align the cleaned DXWR DNA reads to the Nipponbare genome for SV detection. SV detection was conducted using the software SVDetect v0.8b [45]. The pipeline SVFilter was developed in Fei's lab, Cornell University, which can be downloaded from http:// bioinfo.bti.cornell.edu/tool/SVFilter/. This paper is required to be cited to use SVFilter. The parameters for bwa, SVDetect and SVFilter in command lines were provided in Additional file 7. Mapping deletions to genes or QTLs was conducted using in-house Perl programs. Genome graphs (e.g. Fig. 1) were plotted using the software Circos v0.66 [46]. Statistics and plotting were conducted using the software R v2.15.3 with the package ggplot2 [47].

\section{Availability of data and materials}

The NGS data are available in the NCBI SRA database with ID SRP070627. All the supporting data are included as additional files.

\section{Ethics}

Not applicable.

\section{Consent to publish}

Not applicable.

\section{Additional files}

Additional file 1: This file reports 2539 SVs (=links) detected between Dongxiang wild rice and Nipponbare as reference. This file is in the tab delimited format and can be opened using the software Excel. (TXT 1496 kb)

Additional file 2: This file reports mapping the 1568 deletions to rice genes in the reference genome. This file is in the tab delimited format and can be opened using the software Excel. (TXT $268 \mathrm{~kb}$ )

Additional file 3: This file reports mapping the 1568 deletions to QTLS. This file is in the tab delimited format and can be opened using the software Excel. (TXT 1015 kb)

Additional file 4: This file records the relationship between QTLs and rice genes in the same region on the reference chromosome. This file is in the tab delimited format and can be opened using the software Excel. (TXT $2342 \mathrm{~kb}$ )

Additional file 5: This file reports the functional annotation of 99,092 DXWR transcripts from the NCBI NR database using the software blast2go. This file is in the tab delimited format and can be opened using the software Excel. (TXT 12649 kb)

Additional file 6: This file reports 137 KEGG pathways of 4548 DXWR transcripts. This file is in the tab delimited format and can be opened using the software Excel. (TXT $275 \mathrm{~kb}$ )

Additional file 7: Table S1. Eighteen of 1591 Nipponbare-acquired genes. Table S2. Thirteen Nipponbare-acquired genes involving the photosynthesis pathway. Table S3. Eleven Nipponbare-acquired genes involving the oxidative phosphorylation pathway. Table S4. Eighteen DXWR-lost transcripts during domestication. The last section recorded all the parameters used in bwa, SVDetect and SVFilter. (DOC $126 \mathrm{~kb}$ )

\section{Abbreviations}

Chr: chromosome; DXWR: Dongxiang wild rice; GO: Gene Ontology; KEGG: Kyoto Encyclopedia of Genes and Genomes; NGS: Next-Generation Sequencing; NR: Non-Redundant Protein; PK: protein kinase; QTLs: Quantitative Trait Loci; SV: structural variation; TE: transposable element; TF: transcription factor; WAK: wall associated kinase.

\section{Competing interests}

The authors declare that they have no competing interests.

\section{Authors' contributions}

SG and JX conceived and supervised this project. FZ, SY and RC conducted experiments. LM, ZW and XL analyzed the data. SG drafted the main manuscript. TX and XC revised the manuscript and prepared all the figures, tables and additional files. All authors have read and approved the manuscript.

\section{Acknowledgments}

We appreciate the help from Associate Professor Zhangjun Fei in Boyce Thompson Institute for Plant Research, Cornell University and Professor Wenjun Bu in College of Life Sciences, Nankai University. The data analysis in this study was supported by National Scientific Data Sharing Platform for Population and Health Translational Cancer Medicine Specials.

\section{Funding}

This work was supported by grants from National Natural Science Foundation of China (31201191 and 31360327), Natural Science Foundation of Jiangxi Province of China (20132BAB214009 and 20142BAB204012), Special Fund for Agro-scientific Research in the Public Interest (201103007), Key Projects of Jiangxi Education Department (KJLD12059) and Fundamental Research

Funds for the Central Universities. 


\section{Author details}

${ }^{1}$ College of Life Sciences, Jiangxi Normal University, Nanchang, Jiangxi 330022, P. R. China. ${ }^{2}$ College of Animal Science and Veterinary Medicine, Shanxi Agricultural University, Taigu, Shanxi 030801, P. R. China. ${ }^{3}$ Department of Biochemistry and Molecular Biology, College of Medicine, Howard University, Washington DC, WA 20059, USA. ${ }^{4}$ Tianjin Institute of Crop Research, Tianjin Academy of Agricultural Sciences, Tianjin 300381, P. R. China. ${ }^{5}$ College of Life Sciences, Nankai University, Tianjin 300071, P. R. China. ${ }^{6}$ School of Mathematical Sciences, Nankai University, Tianjin 300071, P. R. China. ${ }^{7}$ Tianjin Institute of Agricultural Quality Standard and Testing Technology, Tianjin Academy of Agricultural Sciences, Tianjin 300381, P. R. China.

\section{Received: 14 September 2015 Accepted: 18 April 2016} Published online: 26 April 2016

\section{References}

1. Zhao K, Tung C-W, Eizenga GC, Wright MH, Ali ML, Price AH, et al. Genomewide association mapping reveals a rich genetic architecture of complex traits in Oryza sativa. Nat Commun. 2011;2:467.

2. Oka H-I. Origin of cultivated rice. Philadelphia: Elsevier; 2012.

3. Londo JP, Chiang Y-C, Hung K-H, Chiang T-Y, Schaal BA. Phylogeography of Asian wild rice, Oryza rufipogon, reveals multiple independent domestications of cultivated rice, Oryza sativa. Proc Natl Acad Sci. 2006:103(25):9578-83.

4. Molina J, Sikora M, Garud N, Flowers JM, Rubinstein S, Reynolds A, et al. Molecular evidence for a single evolutionary origin of domesticated rice. Proc Natl Acad Sci. 2011;108(20):8351-6

5. He Z, Zhai W, Wen H, Tang T, Wang Y, Lu X, et al. Two evolutionary histories in the genome of rice: the roles of domestication genes. PLoS Genet. 2011; 7(6):e1002100.

6. Huang X, Kurata N, Wei X, Wang Z-X, Wang A, Zhao Q, et al. A map of rice genome variation reveals the origin of cultivated rice. Nature. 2012;490(7421): 497-501.

7. Gao L, Chen W, Jiang W, Ge S, Hong D, Wang X. Genetic erosion in northern marginal population of the common wild rice Oryza rufipogon Griff. and its conservation, revealed by the change of population genetic structure. Hereditas. 2000;133(1):47-53.

8. $\quad$ Dong YB, Li F, Pei XW, Wang F, Yuan QH, Wu HJ, et al. Genome-wide multilocus analysis of intraspecific differentiation in Oryza rufipogon Griff. from China and the influence of introgression from O. sativa L. Genet Mol Res. 2013;12(4):6103-19.

9. Song ZP, Xu X, Wang B, Chen JK, Lu BR. Genetic diversity in the northernmost Oryza rufipogon populations estimated by SSR markers. TAG Theor Appl Genet. 2003;107(8):1492-9.

10. Luo XJ, Xin XY, Yang JS. Genetic-basis analysis of heterotic loci in Dongxiang common wild rice (Oryza rufipogon Griff.). Genet Res. 2012;94(2):57-61.

11. X-h S, Song Y, Huang R-I, Shan Z, Xiong H-I, Shen L-j. Development of novel cytoplasmic male sterile source from Dongxiang Wild Rice (Oryza rufipogon). Rice Sci. 2013;20(5):379-82.

12. Chen X-R, Yang K-S, Fu J-R, Zhu C-L, Peng X-S, He X-P, et al. Identification and genetic analysis of fertility restoration ability in Dongxiang wild rice (Oryza rufipogon). Rice Sci. 2008;15(1):21-8.

13. Dong $X$, Wang $X$, Zhang L, Yang Z, Xin X, Wu S, et al. Identification and characterization of OsEBS, a gene involved in enhanced plant biomass and spikelet number in rice. Plant Biotechnol J. 2013;11(9):1044-57.

14. He G, Luo X, Tian F, Li K, Zhu Z, Su W, et al. Haplotype variation in structure and expression of a gene cluster associated with a quantitative trait locus for improved yield in rice. Genome Res. 2006;16(5):618-26.

15. Tian F, Li DJ, Fu Q, Zhu ZF, Fu YC, Wang XK, et al. Construction of introgression lines carrying wild rice (Oryza rufipogon Griff.) segments in cultivated rice (Oryza sativa L.) background and characterization of introgressed segments associated with yield-related traits. TAG Theor Appl Genet. 2006;112(3):570-80.

16. Zha X, Luo X, Qian X, He G, Yang M, Li Y, et al. Over-expression of the rice LRK1 gene improves quantitative yield components. Plant Biotechnol J. 2009;7(7):611-20.

17. Liu F, Sun C, Tan L, Fu Y, Li D, Wang X. Identification and mapping of quantitative trait loci controlling cold-tolerance of Chinese common wild rice (O. rufipogon Griff.) at booting to flowering stages. Chin Sci Bull. 2003:48(19):2068-71.

18. Li F, Guo S, Zhao Y, Chen D, Chong K, Xu Y. Overexpression of a homopeptide repeat-containing bHLH protein gene (OrbHLH001) from
Dongxiang Wild Rice confers freezing and salt tolerance in transgenic Arabidopsis. Plant Cell Rep. 2010;29(9):977-86.

19. Xiao N, Huang WN, Li AH, Gao Y, Li YH, Pan CH, et al. Fine mapping of the qLOP2 and qPSR2-1 loci associated with chilling stress tolerance of wild rice seedlings. TAG Theor Appl Genet. 2015;128(1):173-85.

20. Xiao $N$, Huang $W N$, Zhang $X X, G a o ~ Y, L i ~ A H$, Dai $Y$, et al. Fine mapping of qRC10-2, a quantitative trait locus for cold tolerance of rice roots at seedling and mature stages. PLoS One. 2014;9(5):e96046.

21. Zhang X, Zhou S, Fu Y, Su Z, Wang X, Sun C. Identification of a drought tolerant introgression line derived from Dongxiang common wild rice $(\mathrm{O}$. rufipogon Griff.). Plant Mol Biol. 2006;62(1-2):247-59.

22. Springer NM, Ying K, Fu Y, Ji T, Yeh C-T, Jia Y, et al. Maize inbreds exhibit high levels of copy number variation (CNV) and presence/absence variation (PAV) in genome content. PLoS Genet. 2009;5(11):e1000734.

23. McHale LK, Haun WJ, Xu WW, Bhaskar PB, Anderson JE, Hyten DL, et al. Structural variants in the soybean genome localize to clusters of biotic stress-response genes. Plant Physiol. 2012;159(4):1295-308.

24. Feuk L, Carson AR, Scherer SW. Structural variation in the human genome. Nat Rev Genet. 2006;7(2):85-97.

25. Bennetzen JL. Transposable element contributions to plant gene and genome evolution. Plant Mol Biol. 2000;42(1):251-69.

26. Guo X, Bao J, Fan L. Evidence of selectively driven codon usage in rice: implications for GC content evolution of Gramineae genes. Febs Lett. 2007; 581(5):1015-21.

27. Arora R, Agarwal P, Ray S, Singh AK, Singh VP, Tyagi AK, et al. MADS-box gene family in rice: genome-wide identification, organization and expression profiling during reproductive development and stress. BMC Genomics. 2007; 8(1):242

28. Zhang S, Chen C, Li L, Meng L, Singh J, Jiang N, et al. Evolutionary expansion, gene structure, and expression of the rice wall-associated kinase gene family. Plant Physiol. 2005;139(3):1107-24.

29. Krömer S, Stitt M, Heldt HW. Mitochondrial oxidative phosphorylation participating in photosynthetic metabolism of a leaf cell. Febs Lett. 1988; 226(2):352-6.

30. Schwartz A, Zeiger E. Metabolic energy for stomatal opening. Roles of photophosphorylation and oxidative phosphorylation. Planta. 1984;161(2): 129-36.

31. Zhu X-G, Long SP, Ort DR. Improving photosynthetic efficiency for greater yield. Annu Rev Plant Biol. 2010;61:235-61.

32. Makino A. Photosynthesis, grain yield, and nitrogen utilization in rice and wheat. Plant Physiol. 2011;155(1):125-9.

33. Zhang M, Sun H, Fei Z, Zhan F, Gong X, Gao S. Fastq_clean: An optimized pipeline to clean the Illumina sequencing data with quality control. In: Bioinformatics and Biomedicine (BIBM), 2014 IEEE International Conference on: 2014. New York: IEEE; 2014. p. 44-8.

34. Zhou D, Gao S, Wang H, Lei T, Shen J, Gao J, et al. De novo sequencing transcriptome of endemic Gentiana straminea (Gentianaceae) to identify genes involved in the biosynthesis of active ingredients. Gene. 2016;575(1): 160-70.

35. Holl HM, Gao S, Fei Z, Andrews C, Brooks SA. Generation of a de novo transcriptome from equine lamellar tissue. BMC Genomics. 2015;16(1):1.

36. Feder A, Burger J, Gao S, Lewinsohn E, Katzir N, Schaffer AA, et al. A Kelch domain-containing F-box coding gene negatively regulates flavonoid accumulation in Cucumis melo L. Plant Physiol. 2015;169:1714-26.

37. Chen Y-R, Zhong S, Fei Z, Gao S, Zhang S, Li Z, et al. Transcriptome Responses of the Host Trichoplusia ni to Infection by the Baculovirus Autographa californica Multiple Nucleopolyhedrovirus. J Virol. 2014;88(23):13781-97.

38. Xu Y, Gao S, Yang Y, Huang M, Cheng L, Wei Q, et al. Transcriptome sequencing and whole genome expression profiling of chrysanthemum under dehydration stress. BMC Genomics. 2013;14(1):662

39. Guo S, Zhang J, Sun H, Salse J, Lucas WJ, Zhang H, Zheng Y, Mao L, Ren Y, Wang $Z$, et al. The draft genome of watermelon (Citrullus lanatus) and resequencing of 20 diverse accessions. Nat Genet. 2013;45(1):51-8.

40. Li R, Gao S, Hernandez AG, Wechter WP, Fei Z, Ling K-S. Deep sequencing of small RNAs in tomato for virus and viroid identification and strain differentiation. PLoS One. 2012;7(5):e37127.

41. Consortium TTG. The tomato genome sequence provides insights into fleshy fruit evolution. Nature. 2012;485(7400):635-41.

42. Youens-Clark K, Buckler E, Casstevens T, Chen C, DeClerck G, Derwent P, Dharmawardhana $P$, Jaiswal P, Kersey P, Karthikeyan A. Gramene database in 2010: updates and extensions. Nucleic Acids Res. 2010;39(S2):gkq1148. 
43. Friedman N, Grabherr MG, Haas BJ, Yassour M, Levin JZ, Thompson DA, Amit I, Adiconis X, Fan L, Raychowdhury R, et al. Full-length transcriptome assembly from RNA-Seq data without a reference genome. Nat Biotechnol. 2011:29(7):644-52.

44. Langmead B, Trapnell C, Pop M, Salzberg SL. Ultrafast and memory-efficient alignment of short DNA sequences to the human genome. Genome Biol. 2009;10(3):R25

45. Zeitouni B, Boeva V, Janoueix-Lerosey I, Loeillet S, Legoix-Né P, Nicolas A, Delattre O, Barillot E. SVDetect: a tool to identify genomic structural variations from paired-end and mate-pair sequencing data. Bioinformatics. 2010;26(15):1895-6.

46. Krzywinski M, Schein J, Birol I, Connors J, Gascoyne R, Horsman D, Jones SJ, Marra MA. Circos: an information aesthetic for comparative genomics. Genome Res. 2009;19(9):1639-45.

47. Wickham H. ggplot2: elegant graphics for data analysis. Berlin: Springer Science \& Business Media; 2009.

\section{Submit your next manuscript to BioMed Central} and we will help you at every step:

- We accept pre-submission inquiries

- Our selector tool helps you to find the most relevant journal

- We provide round the clock customer support

- Convenient online submission

- Thorough peer review

- Inclusion in PubMed and all major indexing services

- Maximum visibility for your research

Submit your manuscript at www.biomedcentral.com/submit 